\title{
Plea for a holistic approach in stomatology
}

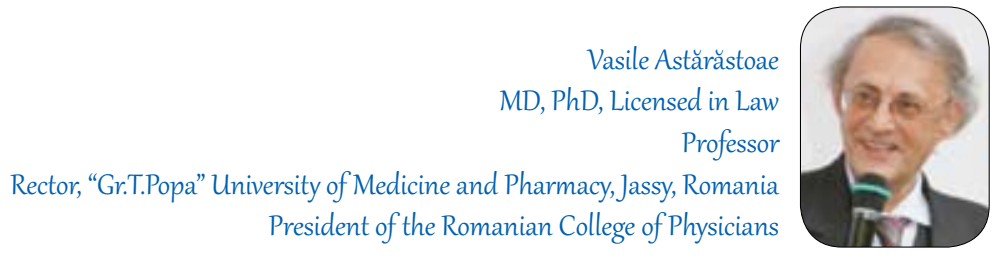

The ethos of the medical profession derives from its tradition. Since the first medical acts, the approach of this profession was anthropological and holistic. This is why, during its evolution, medicine has evolved combining two tendencies: asklepian (knowing) and the samaritan one (feeling compassion for your patient). In the contemporary age, new forces have begun acting: financers influencing health systems while being preoccupied by the rationalization of resources, the importance given to evidence based medicine, guidelines and protocols, subspecialties, the fragmentation of medical care, an excess of technology which removes the human touch (the patient becomes a subject not a person) and, last but not least, the influence of the commercial market which is run by pharmaceutical companies. Slowly, medicine has become institutionalized and instrumentalized, affecting n ot only the classic doctor-patient relationship but also the performances related to protecting people's health.

Among the many branches of medicine, dental medicine (stomatology) emerged as a specialty. One of the many perceptions related to it is that it is a standalone specialty and that only the technical aspects need to be considered. In other words, the stomatologist needs only to be an exquisite technician (professionist) for the dental and buco-maxillary apparatuses.

This approach is not only wrong, but also dangerous for the future of stomatology. It is wrong because contemporary medicine is turning back to a holistic approach and parts of a whole cannot be treated if we do not take into account the interactions between these parts and the whole. Dangerous because those who work in this domain might be considered technicians in the future and not what they are, doctors.

This is why a source for doctors and researchers was needed, one that could show that the medical aspects are well kept in stomatology. This source will be the journal you are reading. This is why a forensic pathologist happily and gratefully accepts to be a part of this beneficial project for all the specialties and doctors out there. 